\title{
The Omnibus Model: A New Model of Data Fusion?
}

\author{
Mark Bedworth and Jane O'Brien \\ Jemity, PO Box 113, Malvern, \\ Worcestershire, WR14 3YJ, UK \\ Email: $\{$ mark.bedworth, jane.obrien\}@ datafusion.clara.net
}

\begin{abstract}
Over the last two decades there have been several process models proposed (and used) for data and information fusion. A common theme of these models is the existence of multiple levels of processing within the data fusion process. In the 1980's three models were adopted: the intelligence cycle, the JDL model and the Boyd control. The 1990's saw the introduction of the Dasarathy model and the Waterfall model. However, each of these models has particular advantages and disadvantages.

A new model for data and information fusion is proposed. This is the Omnibus model, which draws together each of the previous models and their associated advantages whilst managing to overcome some of the disadvantages. Where possible the terminology used within the Omnibus model is aimed at a general user of data fusion technology to allow use by a distributed audience.
\end{abstract}

Keywords: Data fusion architectures and models.

\section{Introduction}

Data fusion is a diverse field encompassing many approaches, algorithms and applications. As with any such complex endeavour the communal knowledge needs to be structured and organised in order to be effective. Over the last two decades several process models have been proposed for data and information fusion [1]. In each case the motivation seems to have been a partitioning of knowledge into sub-tasks since a common theme of these models is the prescription of multiple levels of processing within the data fusion process itself. A multi-disciplinary, international data fusion community is now developing and the issue of standardisation is becoming important. The purpose of this paper is threefold. First we establish the nomenclature for data fusion models, architectures and frameworks. Secondly, we review the main existing data fusion models then identify the advantages and limitations of each. Armed with this information we describe a new model which may be regarded as subsuming the current models. We propose that this model form the basis of a new international standard.

\section{Models, Architectures and Frameworks}

The conceptual organisation of our collected knowledge regarding data fusion has taken many forms. As a result a potential confusion of terminology may arise. We shall therefore define a few terms to describe the way in which data fusion algorithms may be embedded in the context of a larger system.

Three main organisational paradigms are currently in use for describing data fusion systems. These are:

- Models

- Architectures

- Frameworks

We shall describe each of these in turn, highlighting the main differences between them:

Model - we define a model, or more specifically a process model, to be a description of a set of processes. This set of processes should be undertaken before the system may be regarded as fully operational. As such it 
highlights the component functions which the system has but makes no statement regarding their software implementation or physical instantiation. The study of process models forms the main thrust of the remainder of this paper.

Architecture - we define an architecture to be the physical structure of the system. We make particular reference to the way in which data or information is communicated. An architecture includes the arrangement of the component parts, their connectivity and the data flows between the them. The architectural description may be high level - data fusion systems which are described as centralised, hierarchical or distributed are classified by their architecture. It may also be specific - blackboard systems [2] and common object request brokering (CORBA) [3] are specific examples of distributed architectures.

Framework - we define a framework to be a set of axioms and a reasoning system for manipulating entities based on those axioms. As such a framework provides us with a method of inferencing from a data-rich / information-poor source to produce abstract concepts which are information-rich Examples of frameworks currently used in data fusion are probabilistic reasoning, possibilistic reasoning and evidential reasoning.

The remainder of the paper will concentrate on data fusion models. Architectures and frameworks are equally important but are left for future discussions.

\section{Review of Data Fusion Process Models}

Data fusion has its roots in the defence research community of the early 1980's. As a result the first data fusion models were either adapted from existing military oriented process models or were designed with a distinctly military flavour [4]. More recently the use of data fusion has broadened to include industrial, medical and commercial applications. More recent models have acknowledged this migration by reducing the military terminology. However, this still exists to some extent (and needs to be changed).

\subsection{The Intelligence Cycle}

Intelligence processing involves both information processing and information fusion. Although the information is often at a high level, the processes for handling intelligence products are broadly applicable to data fusion in general. There are a number of wellestablished principles of intelligence:

- central control (this avoids the possibility of duplication). The issue of central control is really an architectural issue. It will be seen that avoidance of duplication may be achieved in several ways;

- timeliness (this ensures that the intelligence is available fast enough to be useful);

- $\quad$ systematic exploitation (makes sure that the outputs of the system are used appropriately);

- objectivity (of the sources and the manner in which the information is processed). This is perhaps the factor most relevant to data fusion;

- $\quad$ accessibility (of the information);

- responsiveness (to changing intelligence requirements);

- source protection (to guarantee a source of information with increased longevity);

- continuous review (of the process and the collection strategy).

The UK intelligence community describes the intelligence process as a cycle, which lends itself to modeling the data fusion process [5]. The cycle itself is depicted in Figure 1. Related models exist outside the UK. The British model, unlike the American intelligence model, does not include a specific planning and direction phase that is subsumed in the dissemination process. The UK intelligence cycle comprises four phases:

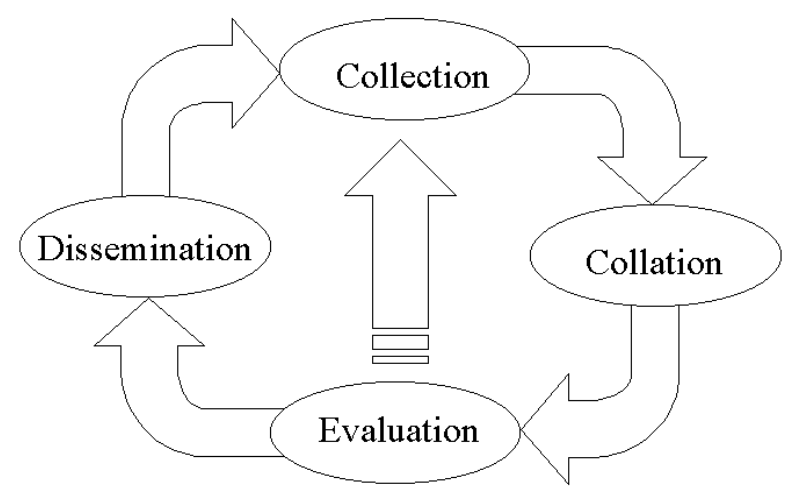

Figure 1: The UK intelligence cycle.

Collection - collection assets such as electronic sensors or human derived sources are deployed to obtain raw 
intelligence data. In the world of intelligence the data is often presented in the form of an intelligence report which is already at a high level of abstraction - either free form text or in a predefined report format.

Collation - associated intelligence reports are correlated and brought together. Some combination or compression may occur at this stage. Collated reports, however, may simply be packaged together ready for fusion at the next stage.

Evaluation - the collated intelligence reports are fused and analysed. Historically, highly skilled human intelligence analysts have undertaken this process. The analysis may identify significant gaps in the intelligence collection. In this case, the analyst may be able to task a collection asset directly. More usually, however, this requirement is included in the disseminated information.

Dissemination - the fused intelligence is distributed to the users (usually military commanders) who use the information to make decisions regarding their own actions and the required deployment of further collection assets.

\subsection{The JDL Model}

In the JDL model, proposed by the US Joint Directors of Laboratories Data Fusion Sub-Group in 1985 [6] and recently updated [7], the processing is divided into five levels as depicted in Figure 2.

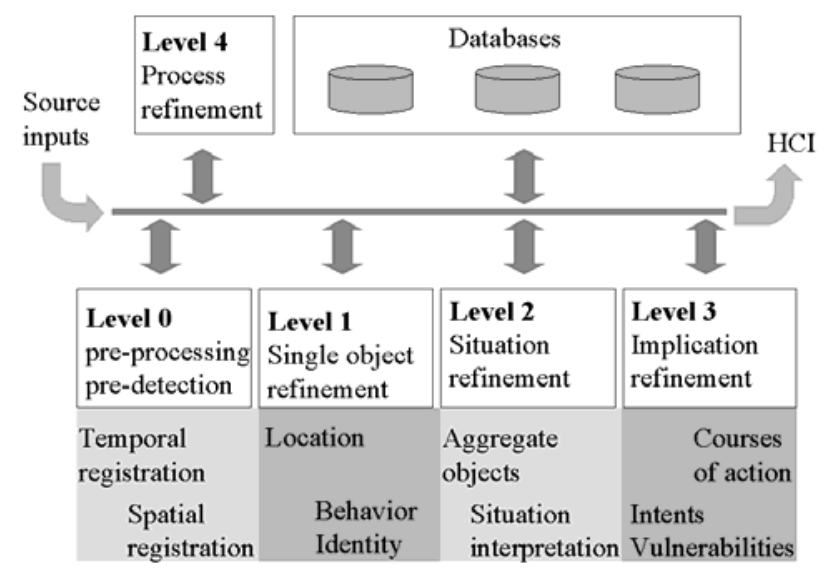

Figure 2: The JDL data fusion process model as recently updated.
Level 0 - sub-object data assessment, is associated with pre-detection activities such as pixel or signal processing, spatial or temporal registration.

Level 1 - object refinement is concerned with the estimation and prediction of continuous (e.g. location or kinematic) or discrete (e.g. behaviour or identity) states of objects.

Level 2 - situation refinement introduces context by examining the relations among entities such as force structure and communication rôles. By aggregating objects into meta-objects an interpretation may be placed on the situation.

Level 3 - implication refinement delineates sets of possible courses of action and the effect they would have in the current situation. This level also introduces the concept that the data fusion system may be operating in an adversarial domain.

Level 4 - process refinement is an element of resource management and used to close the loop by re-tasking resources (e.g. sensors, communications and processing) in order to support the objectives of the mission.

This model has been widely used by the US data fusion community and can now be regarded as the de facto standard for defence data fusion systems, at least in the US. Partly because of its popularity it is applied in a variety of ways [8] and not always used appropriately. The JDL model was never intended to prescribe a strict ordering on the data fusion levels. This was indicated diagrammatically by the use of an information bus rather than a flow structure. Nevertheless, data fusion system designers have consistently assumed this ordering. Clearly there is a need from users to have an ordering whilst the authors of the JDL model rightly defend the need for a model which admits systems of systems with different hierarchies at different levels.

\subsection{The Boyd Control Loop}

The Boyd control loop [9] was first used for modeling the military command process but has since been widely used for data fusion. The Boyd (or OODA) loop possesses four phases as shown in Figure 3. 
The Boyd and JDL models show distinct similarities, although the Boyd model makes the iterative nature of the problem more explicit.

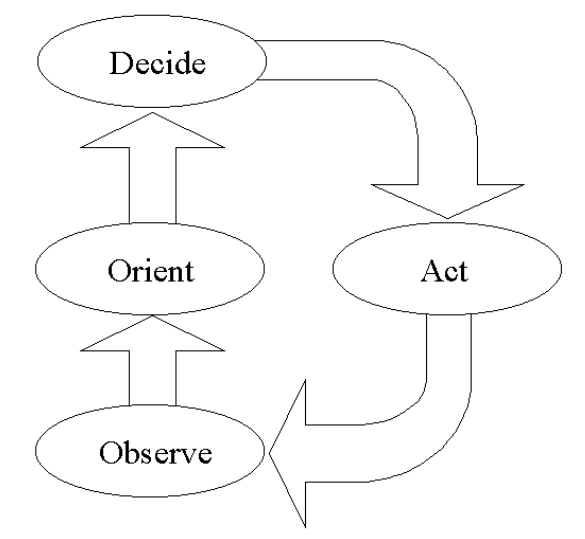

Figure 3: The Boyd (or OODA) loop, which has been used as a data fusion process model.

Observe - broadly comparable to the JDL level 0 and part of the collection phase of the intelligence cycle.

Orient - encompasses the functions of JDL levels 1, 2 and 3. It also includes the structured elements of collection and the collation phases of the intelligence cycle.

Decide - includes JDL level 4 (process refinement and resource management) and the dissemination activities of the intelligence community. It also includes much more (such as logistics and planning).

Act - has no direct analogue in the JDL model and is the only model that explicitly closes the loop by taking account of the effect of decisions in the real world.

The PEMS loop (for Predict, Extract, Match and Search) can be regarded as a perceptual specialisation of the Boyd control loop. The PEMS model has recently attracted attention for automatic target recognition and data fusion [10].

\subsection{The Waterfall Model}

The Waterfall model was proposed in [11] and endorsed by the UK Government Technology Foresight Data Fusion Working Party [12]. It places its main emphasis on the processing functions at the lower levels (see Figure 4). Again, similarities exist with the other models. Sensing and signal processing correspond to JDL level 0 , feature extraction and pattern processing to JDL level 1, situation assessment to JDL level 2 and decision making to JDL level 3. In the Waterfall model the feedback is not explicitly depicted. This appears the major limitation of the Waterfall model which otherwise divides the data fusion process more finely than other models. The Waterfall model has been widely used in the UK defence data fusion community but has not been significantly adopted elsewhere.

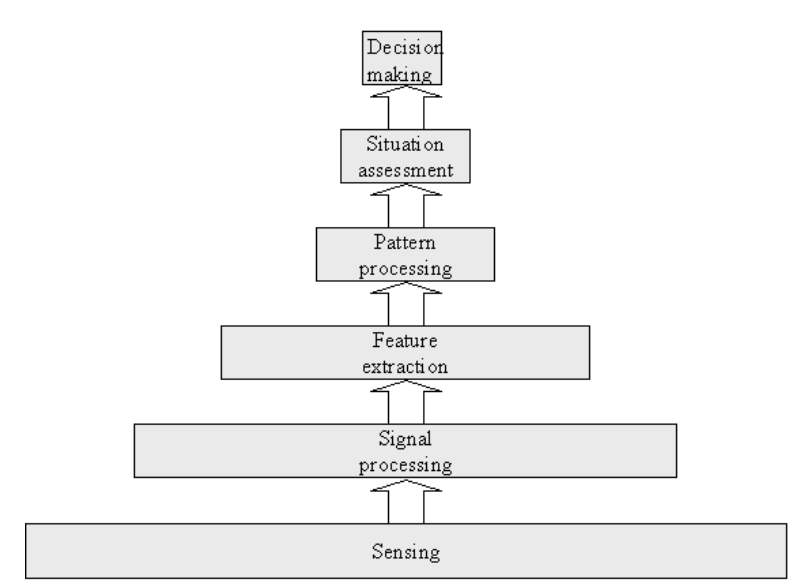

Figure 4: The Waterfall data fusion process model.

\subsection{The Dasarathy Model}

Many researchers have identified the three main levels of abstraction during the data fusion process as being:

- Decisions - symbols or belief values

- Features - or intermediate-level information

- Data - or more specifically sensor data

As was pointed out by Dasarathy in [13], however, fusion may occur both within these levels and as a means of transforming between them. In the model proposed there are five possible categories of fusion as shown in Table 1 at the end of this paper.

\subsection{Model Comparison}

The five models described in the preceding sections can be compared and equivalencies identified where appropriate. Table 2 shows a comparison between the process models described thus far. In some cases the 
equivalence is approximate. Greyed out boxes are not addressed by the specific model. It can be seen that there is some overlap in the way that the different models subdivide the information flow from sensors to actions. The main differences correspond to the amount of detail with which particular processes are represented. This stems from the different uses of the various models and the emphasis they place on certain aspects of the information processing and fusion chain. As can be seen from Table 2, the Waterfall model contains the finest distinction between the lower levels of abstraction, the JDL model at the medium level and the Boyd loop at the higher levels. The intelligence cycle covers all levels but in somewhat compressed detail. The Dasarathy model is based on fusion functions rather than tasks and may therefore usefully be incorporated in each of the fusion activities.

\section{Requirements of a Process Model}

The design and implementation of a data fusion system may usefully be regarded as a problem-solving task. As such a standard approach to problem-solving [14] may be addressed. Of particular relevance are:

- Has the problem been solved before?

- Has the same problem appeared in a different form and is there an existing solution?

- Is there a related problem with similar constraints?

- Is there a related problem with the same unknowns?

- Can the problem be sub-divided into parts that are easier to solve?

- Can the constraints be relaxed to transform the problem into a familiar one?

A process model should facilitate answers to these questions by providing a sub-division of the problem which is rich enough (and detailed enough) to allow reuse of specific knowledge whilst being general enough to allow existing solutions to be deformed into new domains. In this way we may break the goal into subgoals and those into smaller sub-goals until we reach a set of objectives which is attainable $[15,16]$

The model should therefore represent the system under consideration but should also simplify it conceptually. The model will only be of practical use to system developers if this simplification facilitates calculations and predictions.
The foregoing review indicates shortcomings of each of the existing models. Specifically we require a model which:

- defines the ordering of processing;

- makes and cyclic nature of the system explicit;

- admits representation from multiple viewpoints;

- identifies the advantages and limitations of various fusion approaches;

- facilitates the clarification of task-level measures of performance and system-level measures of effectiveness;

- uses a general terminology which is widely accessible;

- does not assume that applications are defence oriented.

With these desires in mind we define a new process model.

\section{The Omnibus Model}

A unified model, to be known as the Omnibus model, is proposed. It comprises a flow chart, a dual-perspective definition and a structured repository of accumulated expertise.

The diagram shown in Figure 5 is based around the cyclic nature of the intelligence cycle and the Boyd control loop but uses the finer definitions of the Waterfall model, each of which can be associated with one of the levels in the JDL and Dasarathy models. In the Omnibus model feedback is explicit and the previously neglected concept of loops within loops is acknowledged. The cyclic nature of the data fusion process is made explicit by retaining the general structure of the Boyd loop. The fidelity of representation expressed by the Waterfall model is then easily incorporated into each of the four main process tasks. The points in the process where fusion may take place are explicitly located. 


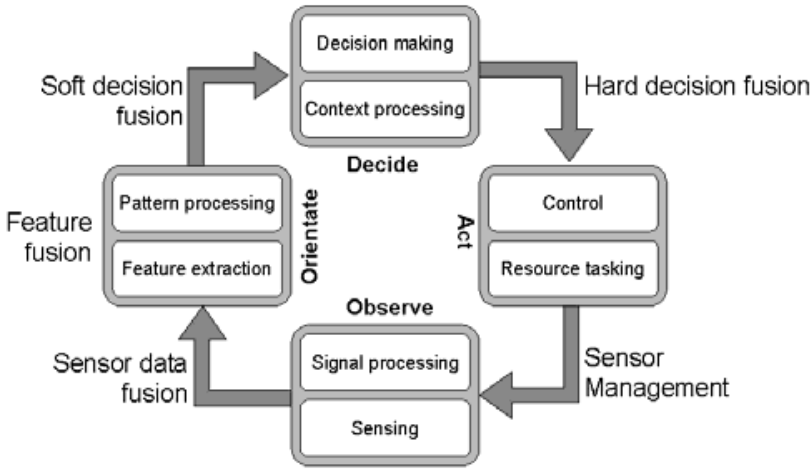

Figure 5: The Omnibus model - a unified data fusion process model.

The model is used in two ways. Firstly, it characterises and sub-divides the overall system aims to provide an ordered list of tasks. Secondly, the same structure may be used to organise the functional objectives of each such task. Using this approach a data fusion solution is categorised using a dual perspective - both by its system aim and its task objective.

The repository of communal knowledge should be structured in a top-down fashion so that analogies can be drawn at either abstract or specific levels. A part of this repository should be a list of advantages and limitations of fusion approaches and techniques. Table 3 shows an example list.

\subsection{Omnibus Case Study}

A technique for fusing multiple detection reports of intruder aircraft is required for embedding within an air defence data fusion system. Using the Omnibus, dualperspective, approach this problem is categorised as:

System aim - orientation (information has been provided by the sources and the aim is to detect aircraft regardless of context)

Task objective - hard decision fusion (the reports contain symbolic data from multi-sensor system).

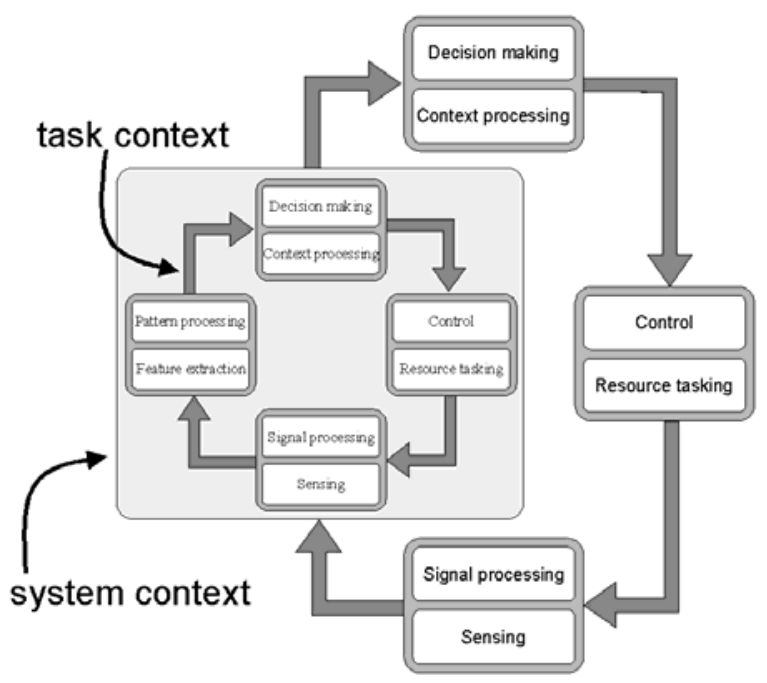

Figure 6: A system within a system representation of the air defence case study example.

This indicates that the system level communication and processing bandwidth will be moderate whilst the tasklevel bandwidth will be low. We may also predict the likely performance at the task level (false alarm rate for example) and begin to analyse its impact on the systemlevel effectiveness (cost effective reduction in own-force casualties for instance).

\section{Conclusions}

A common disadvantage of existing data fusion models is that they are each specifically oriented towards a military domain (to some degree). This is understandable given the origins of data fusion. However, with the increasing use of fusion techniques for industrial and commercial problems it is necessary to define a model with which the extending fusion community is able to identify.

The requirements of a process model have been reexamined and a new model, the Omnibus model, has been proposed. This comprises a process flow chart, a dual-perspective prescription for using it and a structured repository of fusion knowledge. The Omnibus model overcomes some of the main limitations of previous model whilst capitalising on their advantages. The nomenclature used is loosely based on existing notation to maximise familiarity but moves away from a defence-based scheme. 


\section{Recommendations}

The fledgling data fusion community has now reached the maturity that warrants a re-examination of the organisation and structuring of the communal knowledge. The three main categorisation methods models, architectures and frameworks - would lead to a less (unwarranted) duplication and substantial savings from reduced nugatory effort. Some degree of standardisation has to be a good thing in this respect.

It is suggested that over the next 12 months an internationally agreed terminology for (and descriptions of) models, architectures and frameworks be put in place. We observed that several models are currently in use by different parts of the fusion community (largely characterised by their geographic location or their application domain). Some entrenchment of ideas is also evident and the inertia of certain models will make it difficult to inject change.

It was not originally our intention to invent yet another model since we feel there are already too many. However, the Omnibus model emerged as a montage of the best aspects of existing models and therefore represents a unification rather than something new. 


\section{References}

[1] M. Kokar and K. Kim, "Review of Multisensor Data Fusion Architectures", Proceedings of IEEE (1993).

[2] E. Shahbazian, E. Bosse and P. Valin, "Multi-Agent Data Fusion Workstation (MADFW) Architecture", Proceedings of AeroSense conference, SPIE Vol. 3376, pp. 60-68 (1998).

[3] M. Balci and S. Kuru, "A CORBA Based Infrastructure (CORBIS) for Sensor Data Fusion Systems", Proceedings of AeroSense conference, SPIE Vol. 3179, pp. 220-229 (1999).

[4] D. Hall and J. Llinas, "Data Fusion and Multisensor Correlation", Technology Training Corporation course (1985).

[5] A. Shulsky, "Silent Warfare: Understanding the World of Intelliegnce”, Brassey's (1993).

[6] F. White, “ A Model for Data Fusion”, Proceedings $1^{\text {st }}$ National Symposium on Sensor Fusion (1988).

[7] A. Steinberg, C. Bowman and F. White, "Revisions to the JDL Data Fusion Model", Proceedings of AeroSense conference, SPIE Vol. 3719, pp. 430-441 (1999).

[8] L. Klein, "Sensor and Data Fusion Concepts and Applications", SPIE Volume TT14 (1993).

[9] J. Boyd, "A Discourse on Winning and Losing", Maxwell AFB lecture, (1987).

[10] J. Gainey and E. Blasch, "Development of Emergent Processing Loops as a System of Systems Concept", Proceedings of AeroSense conference, SPIE Vol. 3179, pp. 186-195 (1999).

[11] M. Bedworth, "Probability Moderation for Multilevel Information Processing", DRA Technical Report DRA/CIS(SE1)/651/8/M94.AS03BP032/1 (1994).

[12] M. Markin, C. Harris, M. Bernhardt, J. Austin, M. Bedworth, P. Greenway, R. Johnston, A. Little and D. Lowe, "Technology Foresight on Data Fusion and Data Processing", Publication of The Royal Aeronautical Society, (1997).
[13] B. Dasarathy, "Sensor Fusion Potential Exploitation - Innovative Architectures and Illustrative Applications", Proceedings of IEEE, Volume 85, Number 1, pp 24-38 (1997).

[14] G. Polya, "How to Solve It", ???? (1945).

[15] R. Atkinson, R. Atkinson, E. Smith, D. Bem and E. Hilgard, "Introduction to Psychology", Harcourt Brace Jovanovich (1990).

[16] J. Anderson, "Cognitive Psychology and its Implications”, New York Freeman (1985). 


\begin{tabular}{|l|l|l|l|}
\hline Input & Output & Notation & Analogues \\
\hline Data & Data & DAI-DAO & Data-level fusion \\
\hline Data & Features & DAI-FEO & Feature selection and feature extraction \\
\hline Features & Features & FEI-FEO & Feature-level fusion \\
\hline Features & Decisions & FEI-DEO & Pattern recognition and pattern processing \\
\hline Decisions & Decisions & DEI-DEO & Decision-level fusion \\
\hline
\end{tabular}

Table 1: The five levels of fusion in the Dasarathy model.

\begin{tabular}{|c|c|c|c|c|}
\hline $\begin{array}{ll}\text { Activity } & \text { being } \\
\text { undertaken } & \end{array}$ & Waterfall model & JDL model & Boyd Loop & Intelligence Cycle \\
\hline $\begin{array}{l}\text { Command } \\
\text { execution }\end{array}$ & & & Act & \multirow[t]{2}{*}{ Disseminate } \\
\hline $\begin{array}{l}\text { Decision making } \\
\text { process }\end{array}$ & Decision making & Level 4 & Decide & \\
\hline Threat assessment & & Level 3 & \multirow{4}{*}{ Orient } & \multirow[b]{2}{*}{ Evaluate } \\
\hline $\begin{array}{l}\text { Situation } \\
\text { assessment }\end{array}$ & $\begin{array}{l}\text { Situation } \\
\text { assessment }\end{array}$ & Level 2 & & \\
\hline \multirow[t]{2}{*}{$\begin{array}{l}\text { Information } \\
\text { processing }\end{array}$} & Pattern processing & \multirow{2}{*}{ Level 1} & & \multirow{3}{*}{ Collate } \\
\hline & Feature extraction & & & \\
\hline Signal processing & Signal processing & Level 0 & \multirow[t]{2}{*}{ Observe } & \\
\hline $\begin{array}{l}\text { Source / sensor } \\
\text { acquisition }\end{array}$ & Sensing & & & Collect \\
\hline
\end{tabular}

Table 2: A comparison between the data fusion process models described in this paper.

\begin{tabular}{|l|l|l|l|l|}
\hline Fusion level & Bandwidth & Performance & Advantages & Limitations \\
\hline Hard decisions & Very low & Depends on system & $\begin{array}{l}\text { Simplicity for large } \\
\text { systems }\end{array}$ & $\begin{array}{l}\text { Poor performance } \\
\text { for small systems }\end{array}$ \\
\hline Soft decisions & Low & Often good & $\begin{array}{l}\text { Bandwidth / } \\
\text { performance trade- } \\
\text { off }\end{array}$ & $\begin{array}{l}\text { Sophisticated } \\
\text { algorithms needed } \\
\text { for correlated } \\
\text { sources }\end{array}$ \\
\hline Features & Moderate & Good $\rightarrow$ high & High performance & $\begin{array}{l}\text { Difficult to select } \\
\text { correct features }\end{array}$ \\
\hline Data & High $\rightarrow$ very high & Potentially optimal & $\begin{array}{l}\text { Possibility of using } \\
\text { physical models }\end{array}$ & $\begin{array}{l}\text { High bandwidth } \\
\text { restricts use to } \\
\text { single platform } \\
\text { systems }\end{array}$ \\
\hline
\end{tabular}

Table 3: The advantages and disadvantages of the four levels of data fusion. 